\title{
Serological Diagnosis of Helicobacter pylori Infection in Patients With a Polycystic Ovary Syndrome
}

\author{
Amir Hossein Kiani ${ }^{1}$; Elham Asadbeik ${ }^{2}$; Meysam Hasannejad Bibalan ${ }^{3}$; Mansour Sedighi ${ }^{3}$; \\ Morteza Eshaghi ${ }^{3}$; Mehrdad Gholami ${ }^{3}$; Abazar Pournajaf ${ }^{3, *}$ \\ ${ }_{1}^{1}$ Department of Immunology, Faculty of Medicine, Jundishapur University of Medical Sciences, Ahvaz, IR Iran \\ ${ }^{2}$ Department of Biotechnology, Faculty of Medicine, Shahrekord University of Medical Sciences, Shahrekord, IR Iran \\ ${ }^{3}$ Department of Microbiology, Faculty of Medicine, Iran University of Medical Sciences, Tehran, IR Iran \\ ${ }^{*}$ Corresponding author: Abazar Pournajaf, Department of Microbiology, Faculty of Medicine, Iran University of Medical Sciences, Tehran, IR Iran. Tel/Fax: +98-2188058649, \\ E-mail:abazar_pournajaf@yahoo.com
}

Received: January 27, 2015; Revised: March 3, 2015; Accepted: March 15, 2015

\begin{abstract}
Background: Polycystic Ovary Syndrome(PCOS) is the most common endocrine disorder in $4 \%$ - $6 \%$ of women in the reproductive age and is a common cause of infertility. Even though the number of investigations is scarce, studies show that Helicobacter pylori infection may influence reproduction.

Objectives: The purpose of this study was to determine and compare the levels of $H$. pylori specific antibodies IgA, IgG and anti-CagA at both PCOS and non-PCOS women with their spouses using the serological test.

Patients and Methods: In this cross-sectional study, 127 women with their spouses (age range, 30 - 60 years) were selected. These patient were referred to infertility center of Shariati Hospital in Tehran, Iran, with a diagnostic criteria of PCOS based on Androgen Excess Society (AES). The specific antibodies of IgA, IgG and anti-CagA were measured using the commercial Enzyme-Linked Immunosorbent Assay (ELISA) kit.

Results: The positive titers of H.pyloriantibodies IgA, IgG and anti-CagAin the PCOS group were 45(35\%), 79(62\%)and 77(60.5\%), respectively, while in non-PCOS group were $38(30 \%), 76(60 \%)$ and 50 (39.5\%), respectively. The sera positive for IgA, IgG and anti-CagA antibodies in spouses of the non-PCOS group were 38 (30\%), 84 (66\%) and 79 (62\%) respectively, but in spouses of the PCOS group were $51(40 \%), 83(66 \%)$ and 48 (38\%), respectively. The results showed that $H$. pylori infection probably did not affect infertility or reproduction.

Conclusions: Findings of this study demonstrate no significant difference between levels of $H$. pylori specific antibodies of IgA, IgG, anti-CagA and the presence of PCOS disorders, and also indicate that serologic testing is a sensitive method for the detection of $H$. pylori antibodies. The high prevalence of $\mathrm{H}$. pylori positive antibody levels in both PCOS and non-PCOS patients can be probably associated with the high frequency of H. pylori infection.
\end{abstract}

Keywords: Helicobacter pylori; Serological Tests; Polycystic Ovary Syndrome

\section{Background}

Polycystic Ovary Syndrome (PCOS), is the most common endocrine disorder in $4 \%$ - $6 \%$ women of reproductive age and a common cause of infertility (1). In the recent years, studies have shown the role of Helicobacter pylori in the development of endocrinopathies. Yavasoglu et al. reported a raised incidence of $H$. pylori seropositivity in women with PCOS (2). Helicobacter pylori infection affects more than half of the world's people and is most commonly associated with chronic gastritis which afterward increases the risk of many serious complications including gastric cancer (3). In addition, there are limited reports showing potential association between infertility and $H$. pylori infection (4). Polycystic ovary syndrome is a condition, previously known as Stein-Leventhal syndrome, that is characterized by multiple ovarian cysts together with oligomenorrhoea (scanty periods) or amenorrhea (absence of periods), reduced fertility, hirsutism (excessive hairiness), and obesity
(5-7). Most women with PCOS begin menstruation at a normal age, but between the ages of 15 - 30 years the periods become irregular and then stop. In addition, they often become infertileand obese, and develop excess facial hair (Hirsutism), acne and male balding-patches. Several factors contribute to the pathogenesis of illness, including insulin resistance, impaired secretion of surgery, genetics, environmental chemical pollution, food additives and chronic inflammation (8). The prevalence of $H$. pylori infection is varied and related to age, geographical location and socioeconomic status (9). The prevalence of $H$. pylori seems to be more rapid in developing than developed countries. Helicobacterpylori acquisition in developing countries may up to $70 \%$ compared to $40 \%$ or less in developed countries. The results may be related to improvements in hygiene conditions (10). The cag Pathogenicity Island (cag PAI) is a 40-kb DNA insertion element which encodes CagA. The

Copyright (C) 2015, Infectious Diseases and Tropical Medicine Research Center. This is an open-access article distributed under the terms of the Creative Commons Attribution-NonCommercial 4.0 International License (http://creativecommons.org/licenses/by-nc/4.0/) which permits copy and redistribute the material just in noncommercial usages, provided the original work is properly cited. 
gene is present in approximately 50\% - 60\% of $\mathrm{H}$. pylori isolates, but not present in all strains (11). Detection of a serological response may therefore give clinically relevant information about the infecting strain (12). Because of white blood cells involvement against H. pylori, IgA and IgG titers rise in serum. Some researchers have reported that $H$. pylori strains may be colonized in stomach-vagina cells and may be causing inflammation, asymptomatic infection and then infertility.

\section{Objectives}

The purpose of this study was to determine and compare the levels of $H$. pylori specific antibodies of IgA, IgG and anti-CagA at both PCOS and non-PCOS women with their spouses using the serological test.

\section{Patients and Methods}

This cross-sectional study was conducted on 127 women with their spouses (age range, 30 - 60 years old) from June 2011 to April 2012 in Tehran, Iran. These patients were referred to infertility center of Shariati Hospital in Tehran, Iran, with a diagnostic criteria of PCOS based on Androgen Excess Society (AES) (13). This previous prospective study (2) comprised 127 women with PCOS, who were diagnosed using the revised diagnostic criteria of the European Society for Human Reproduction and Embryology and the American Society for Reproductive Medicine (ESHRE/ ASRM) 2003. Some of the important criteria for PCOS in the present study were based on the pattern of the menstrual cycle (menstrual cycle intervals $<35$ ), hirsutism, acne and androgenic alopecia (with grades $>8$ ) based upon criteria Ferriman-Gallwey (mFG) score and detection of ultrasound by gynecologists. The control group consisted of 127 women (age range, 30 - 60 years old) without PCOS, which were admitted to the infertility center of Shariati Hospital in Tehran, Iran. Exclusion criteria for both groups, including patients with adrenal hyperplasia, Cushing's syndrome, hyperprolactinemia, androgenproducing tumors, treatment of $H$. pylori eradication and the use of antibiotics during the past year. After obtaining an informed consent from both groups and their spouses, a venous blood sample (4- $6 \mathrm{~mL}$ ) was taken for serology tests. Centrifugation at 4000 RPM for 5 minutes was used to separate the serum and for the study of $H$. pylori antibodies IgA by kit (Bio RAD, USA, cat No.; 25225), using the Enzyme-Linked Immunosorbent Assay. Sensitivity and specificity of ELISA were $84 \%$ and $97 \%$, respectively. Cut-off antibody levels according to the instructions were $\geq 1.0 \mathrm{U} /$ $\mathrm{mL}$ as positive, between $0.89-0.99 \mathrm{U} / \mathrm{mL}$ as equivocal and $<$ $0.89 \mathrm{U} / \mathrm{mL}$ were considered as negative. The IgG assay was performed (Bio-Rad, Hercules, CA, USA) with sensitivity and specificity greater than $98 \%$. Interpretation of the cutoff value of IgG antibody is similar to IgA antibody. Serum anti-CagA IgG antibody levels were also evaluated by ELISA method using commercial kits (Diagnostic Bioprobes, Italy). The serum concentrations of anti-CagA antibodies were expressed in arbitrary units per milliliter (Uarb/mL) as no International Standard is available. According to the manufacturer's guidelines, the value of 5 Uarb/mL was used to discriminate the negative samples from positive ones. Demographic data of both studied groups (case and control) with antibody titer were analyzed using KruskalWallis, Mann-Whitney U-test, chi-square and Fisher's exact tests as appropriate and P-values less than 0.05 were considered as statistically significant.

\section{Results}

In this study, 254 women were studied in two groups, PCOS $(n=127)$ and non-PCOS $(n=127)$. The mean age was $36.13 \pm 3.45$ in PCOS women and $35.84 \pm 3.58$ in non-PCOS women. In the two studied groups of PCOS and non-PCOS, no statistically significant difference was observed in the mean age of the spouses of the non-PCOS and PCOS groups $(P=0.20)$ (Table 1$)$. No significant difference was observed in demographic characteristics (women's educational status, occupation, educational status and occupation of the spouses) between the two groups $(\mathrm{P}>0.05)$. The prevalence of increased levels of IgG antibodies to $H$. pylori in the PCOS patients was $79(62 \%)$ at a cut-off point of $\geq 1.0 \mathrm{U} / \mathrm{mL}$ as positive, while 48 (38\%) at a cut-off point of $0.89-0.99$ or $<0.89 \mathrm{U} / \mathrm{mL}$ were as equivocal or negative. Seventy-six patients (60\%) were sera-positive for the IgG antibody to $H$. pylori at non-PCOS group while 51 cases were seronegative, giving a $40 \%$ sera-prevalence level of anti $H$. pylori IgG from non-PCOS group. From a total of 127 PCOS women tested for H. pylori IgA antibody, 45 cases (35\%) were positive for IgA antibodies, while 38 cases (30\%) were positive in non-PCOS women. The overall seroprevalence of positive anti-CagA IgG antibodies in PCOS women was $77(60.5 \%)$ and in non-PCOS women were 50 (39.5\%). The prevalence rates of serum anti-CagA antibody were $60.5 \%$ and $39.5 \%$ in infected $H$. pylori PCOS women and nonPCOS women with mean titers of $67.4 \pm 68.54$ Uarb/mL and $32.9 \pm 35.73 \mathrm{Uarb} / \mathrm{mL}$, respectively. The sera positive for IgA, IgG and anti-cagA antibodies among the spouses of the non-PCOS group were 38 (30\%), 84 (66\%) and $79(62 \%)$ respectively, but in spouses of the PCOS group were 51 (40\%), $83(66 \%)$ and 48 (38\%), respectively. The mean titer of antiCagA IgG in spouses of the non-PCOS group was $66.43 \pm$ 67.21 Uarb/mL and in the spouses of the PCOS group was $31.56 \pm 33.42 \mathrm{Uarb} / \mathrm{mL}$. There was no significant difference regarding the prevalence of serum anti-CagA antibodies between the PCOS and non-PCOS groups with their spouses; however, this parameter was higher in PCOS patients with their spouses than in non-PCOS group with their spouses. Results of this study showed no significant difference in a positive serum titer of $H$. pylori specific antibodies IgA, IgG and anti-CagA between the PCOS and non-PCOS groups and also in their spouses $(P>0.05)$ (Table 2$)$. These findings showed that $H$. pylori infection probably did not affect infertility or reproduction in people and also high levels of $H$. pylori specific antibodies were not associated with a PCOS disorder. 
Kiani AH et al.

\begin{tabular}{|c|c|c|c|}
\hline Criteria & PCOS & Non-PCOS & P Value \\
\hline \multicolumn{4}{|l|}{ Woman's job } \\
\hline Employed & $10(8)$ & $12(9)$ & 0.75 \\
\hline Housewife & $117(92)$ & $115(91)$ & \\
\hline \multicolumn{4}{|l|}{ Women's education } \\
\hline Not educated & $65(51)$ & $67(53)$ & 0.10 \\
\hline Educated & $62(49)$ & $60(47)$ & \\
\hline \multicolumn{4}{|l|}{ Spouse's education } \\
\hline Not educated & $121(95)$ & $118(93)$ & 0.13 \\
\hline Non-Employed & $6(5)$ & $9(7)$ & \\
\hline \multicolumn{4}{|l|}{ Men's job } \\
\hline Unemployed & $3(2)$ & $8(6)$ & \\
\hline Workers & $76(60)$ & $46(36)$ & \\
\hline Employee & $23(18)$ & $26(20)$ & \\
\hline Other & $25(20)$ & $47(38)$ & \\
\hline The mean age of women & $36.13 \pm 3.45$ & $35.84 \pm 3.58$ & 0.20 \\
\hline The mean age of men & $34.69 \pm 5.76$ & $35.12 \pm 4.86$ & 0.11 \\
\hline
\end{tabular}

Table 2. Evaluation of Antibodies (IgG, IgA and IgG anti-CagA) Against Helicobacter pylori in Both Polycystic Ovary Syndrome and Non-Polycystic Ovary Syndrome Groups and Their Spouses ${ }^{\mathrm{a}}$, b

\begin{tabular}{|c|c|c|c|c|c|c|}
\hline & PCOS Group & Non-PCOS Group & PValue & $\begin{array}{c}\text { Spouses of PCOS } \\
\text { Group }\end{array}$ & $\begin{array}{c}\text { Spouses of Non-PCOS } \\
\text { Group }\end{array}$ & PValue \\
\hline IgA, level of Serum & & & 0.42 & & & 0.14 \\
\hline Positive & $45(35)$ & $38(30)$ & & $51(40)$ & $38(30)$ & \\
\hline Negative & $82(65)$ & $89(70)$ & & $76(60)$ & $89(70)$ & \\
\hline IgG, level of Serum & & & 0.84 & & & 0.25 \\
\hline Positive & $79(62)$ & $76(60)$ & & $83(66)$ & $84(66)$ & \\
\hline Negative & $48(38)$ & $51(40)$ & & $44(34)$ & $43(34)$ & \\
\hline Anti-CagA Seropositivity, Uarb/mL & & & 0.82 & & & 0.21 \\
\hline Positive & $77(60.5)$ & $50(39.5)$ & & $48(38)$ & $79(62)$ & \\
\hline Negative & $50(39.5)$ & $77(60.5)$ & & $79(62)$ & $48(38)$ & \\
\hline
\end{tabular}

${ }^{\mathrm{a}}$ Abbreviation: PCOS, polycystic ovary syndrome.

${ }^{b}$ Data are presented as No. (\%).

\section{Discussion}

Polycystic ovary syndrome has been associated with an increased hazard of miscarriage, but particular mechanisms related to this observation remain unknown. This infection is more common in individuals with infertility (14). Infected women have anti-H. pylori antibodies in cervical mucus and follicular fluid that may decrease sperm motility and cross-react immunologically with spermatozoa, conceivably hampering the oocyte/sperm fusion. Infection by CagA positive $H$. pylori enhances the risk of preeclampsia, which is a main cause of fetus death (15). The relationship between infectious agents such as $H$. pylori infection and reproductive performance in recent years was considered (4). Figura evaluated the level of serum antibodies in 167 infertile patients and 837 patients in the control group, that results showed the rate of infection is significantly higher in the infertile group and thus, H. pylori infection can lead to the possible problems associated with infertility in both males and females (16). In association with PCOS and H. pylori infection, Yavasoglu conducted the first study on 35 men and 50 women with non-PCOS and PCOS groups with evaluation of antibodies IgG. In this study, the positive IgG at female cases 
with PCOS was $40 \%$ and in control group was $22 \%$,which showed a significant difference (2). In a study of Jafarzadeh et al. (17), 386 children aged 1 - 15 years and 200 adults aged 20 - 60 years were investigated in specific serum immunoglobulin G to H. pylori and CagA. They were reported the seroprevalence of IgG antibody against $H$. pylori in adults that was significantly higher than that observed in children (67.5\% vs 46.6\%; P < 0.000003). Moreover, they indicated the prevalence of serum anti-CagA antibody that was $72.8 \%$ in infected children and $67.4 \%$ in adults. In addition, they were claimed that the mean titer of serum anti-CagA antibodies was significantly higher among children in comparison to the adult (64.1 Uarb/mL vs 30.7; $\mathrm{P}<0.03$ ) (17). In the present study, positive IgG antibody against $H$. pylori in women with PCOS was $79(62 \%)$ and in women without PCOS was 76 (60-\%). The positive titers of IgA antibody in women with and without PCOS were 45 (35\%) and those 38 (30\%), respectively. The titers of serum anti-CagA antibody were $60.5 \%$ and $39.5 \%$ in infected $H$. pylori women with PCOS and non-PCOS group with mean titers of $67.4 \pm 68.54 \mathrm{Uarb} / \mathrm{mL}$ and $32.9 \pm 35.73 \mathrm{Uarb} / \mathrm{mL}$, respectively. The sera positive IgA and IgG in PCOS women with their spouses were higher than non-PCOS women with their spouses, but the result of anti-CagA antibody was vice versa in these women and their spouses. No statistically significant difference was seen between the two groups, PCOS women and non-PCOS women with their spouses. Moreover, Yavasoglu reported the percentage of positive IgG titers in $60 \%$ of women with PCOS and $40 \%$ of women without PCOS (2). Due to the lack of a significant statistical association between positive cases IgG antibodies against H. pylori in the two groups in this study, there is no statistically significant association between Iranian men and women; perhaps there is a higher rate of $H$. pylori infection. Here, we report the concerning evidences of decrease in reproductive potential occurring in individuals infected by $\mathrm{H}$. pylori, especially by strains expressing CagA. Thus, probably comprehensiveness of the disease in developing countries (70-90\%), due to the lack of statistical difference between the PCOS and nonPCOS women is common. Also, no significant difference was observed in levels of $H$. pylori seropositivity between the two groups (with and without PCOS). These results showed that anti-H. pylori and anti-CagA antibodies were common in the PCOS patients. The results demonstrate no significant difference between levels of $H$. pylori specific antibodies (IgA, IgG and anti-CagA) and the presence of PCOS disorders and the results were almost similar in the two groups (PCOS and non-PCOS whether in women or in their spouses). The results of this study indicate that serological test is a sensitive method for the detection of H. pylori specific antibodies of IgA, IgG and anti-CagA. The high prevalence of $H$. pylori antibody positive levels in both PCOS and non-PCOS patients can be probably associated with the high frequency of $H$. pylori infection.

\section{Acknowledgements}

We would like to appreciate Dr. Gholamreza Irajian and Dr. Abdolah Ardebili for their technical assistance.

\section{References}

1. Carmina E, Lobo RA. Polycystic ovary syndrome (PCOS): arguably the most common endocrinopathy is associated with significant morbidity in women. J Clin Endocrinol Metab. 1999;84(6):1897-9.

2. Yavasoglu I, Kucuk M, Cildag B, Arslan E, Gok M, Kafkas S. A novel association between polycystic ovary syndrome and Helicobacter pylori. Am J Med Sci. 2009;338(3):174-7.

3. Kusters JG, van Vliet AH, Kuipers EJ. Pathogenesis of Helicobacter pylori infection. Clin Microbiol Rev. 2006;19(3):449-90.

4. Hajishafiha M, Ghasemi-Rad M, Memari A, Naji S, Mladkova N, Saeedi V. Effect of Helicobacter pylori infection on pregnancy rates and early pregnancy loss after intracytoplasmic sperm injection. Int J Womens Health. 2011;3:329-35.

5. Ehrmann DA. Polycystic ovary syndrome. $N$ Engl J Med. 2005;352(12):1223-36

6. Gambineri A, Pelusi C, Vicennati V, Pagotto U, Pasquali R. Obesity and the polycystic ovary syndrome. Int J Obes Relat Metab Disord. 2002;26(7):883-96.

7. Teede H, Deeks A, Moran L. Polycystic ovary syndrome: a complex condition with psychological, reproductive and metabolic manifestations that impacts on health across the lifespan. BMC Med. 2010;8:41.

8. Solomon CG. The epidemiology of polycystic ovary syndrome. Prevalence and associated disease risks. Endocrinol Metab Clin North Am. 1999;28(2):247-63.

9. Torres J, Perez-Perez G, Goodman KJ, Atherton JC, Gold BD, Harris PR, et al. A comprehensive review of the natural history of Helicobacter pylori infection in children. Arch Med Res. 2000;31(5):431-69.

10. Salih BA. Helicobacter pylori infection in developing countries: the burden for how long? Saudi J Gastroenterol. 2009;15(3):201-7.

11. Blaser MJ, Perez-Perez GI, Kleanthous H, Cover TL, Peek RM, Chyou $\mathrm{PH}$, et al. Infection with Helicobacter pylori strains possessing cagA is associated with an increased risk of developing adenocarcinoma of the stomach. Cancer Res. 1995;55(10):2111-5.

12. Mitchell HM, Hazell SL, Li YY, Hu PJ. Serological response to specific Helicobacter pylori antigens: antibody against CagA antigen is not predictive of gastric cancer in a developing country. Am J Gastroenterol.1996;91(9):1785-8.

13. Wild RA, Carmina E, Diamanti-Kandarakis E, Dokras A, EscobarMorreale HF, Futterweit W, et al. Assessment of cardiovascular risk and prevention of cardiovascular disease in women with the polycystic ovary syndrome: a consensus statement by the Androgen Excess and Polycystic Ovary Syndrome (AE-PCOS) Society. $J$ Clin Endocrinol Metab. 2010;95(5):2038-49.

14. Futterweit W. Polycystic ovary syndrome: clinical perspectives and management. Obstet Gynecol Surv. 1999;54(6):403-13.

15. Ambrosini G, Andrisani A, Fiore C, Faggian D, D'Antona D, Ragazzi E, et al. Anti-Helicobacter pylori antibodies in cervical mucus: a new cause of infertility. Eur J Obstet Gynecol Reprod Biol. 2011;155(2):157-60.

16. Figura N, Piomboni P, Ponzetto A, Gambera L, Lenzi C, Vaira D. Presence of anti Helicobacter pylori antibodies in follicular liquid, sperm and vaginal mucus samples of infected patients with fertility disorders.Strasbourg Workshop; 2001.

17. Jafarzadeh A, Rezayati MT, Nemati M. Specific serum immunoglobulin $\mathrm{G}$ to $\mathrm{H}$ pylori and CagA in healthy children and adults (south-east of Iran). World J Gastroenterol. 20 07;13(22):3117-21. 